\title{
Informationssysteme in der Finanzwirtschaft
}

von

Hans Ulrich Buhl, Christof Weinhardt 
Auf dem Weg ins neue Jahrhundert sehen sich Finanzdienstleister einem wettbewerbsintensiven, technologisch geprägten Umfeld und dynamischen, globalen Märkten gegenüber. Offensive Strategien und deren konsequente Umsetzung auf Basis moderner Informations- und Kommunikationssysteme sind zwingend notwendig. Innovationsfähigkeit, Flexibilität sowie die Nutzung der Chancen, die neue Informationstechnologien bieten, sind Schlüsselfaktoren zum Erfolg.

In diesem Kontext müssen insbesondere die strategischen Aspekte und Auswirkungen von Internet- und Intranet-Technologien Berücksichtigung finden. Angesichts einer breiten Akzeptanz von Informations- und Kommunikationssystemen auch im Privatkundenbereich reicht die reine Präsenz im und die Informationsübermittlung über das Internet nicht mehr aus. Vielmehr sind neue Geschäftskonzepte erforderlich, die auch die Durchführung von Transaktionen über dieses Medium umfassen.

Dies betrifft nicht nur Finanzdienstleister i. e. S., sondern auch neue Finanzintermediäre und insbesondere die Börsen. Die Implementierung elektronischer Marktplätze mit weltweiten, dezentralen $\mathrm{Zu}$ gangsmöglichkeiten beeinflußt nachhaltig die bestehenden nationalen Börsenstrukturen. Der Weg von den traditionellen Börsenplätzen hin zu Börsennetzen ist unumkehrbar. Die konkrete Ausgestaltung dieser Netze und die Rolle des Internet für den Wertpapierhandel stehen im Zentrum des Interesses.

Darüber hinaus müssen Finanzdienstleister angesichts volatiler Märkte, wachsender Bedeutung derivativer Finanzinstrumente und strenger regulatorischer Anforderungen ein aktives Ertrags- und Risikomanagement betreiben, um auch in Zeiten von Disintermediation und Securitization im Wettbewerb bestehen zu können.

Nach dem Erfolg der IF'95 in München wurde mit der IF-98 - Informationssysteme in der Finanzwirtschaft erneut ein Forum für Praktiker und Wissenschaftler geschaffen, um die oben genannten Fragestellungen an der Schnittstelle zwischen Informationstechnik und Finanzwirtschaft diskutieren zu können.

Diese Tagung fand am 7. und 8. Oktober im Communication Center Eschborn (CCE) der Deutschen Bank AG sowie im Hause der Deutschen Börse AG statt und wurde in Zusammenarbeit mit der GI-

\section{Informationssysteme in der Finanzwirtschaft}

\section{Hans Ulrich Buhl, Christof Weinhardt}

Fachgruppe „Informationssysteme in der Finanzwirtschaft" unter der Leitung von Christof Weinhardt, Universität Gießen, und seinem Team veranstaltet.

Mit einer guten Mischung der Beiträge, aber auch einem nahezu ausgewogenen Verhältnis von Teilnehmern aus Praxis und Wissenschaft konnte ein wesentliches Ziel der Tagung erreicht werden, nämlich die Kluft zwischen Wissenschaft und Praxis etwas weiter zu schließen.

Die Herausgeber der WIRTSCHAFTSINFORMATIK haben beschlossen, mit diesem Schwerpunktheft herausragende Arbeiten dieser Tagung ihren Lesern zugänglich zu machen. Aus einer Menge von mehr als 60 Beiträgen wurden im ReviewProzeß insgesamt 32 Arbeiten ausgewählt und im Rahmen von drei Sektionen zu einem attraktiven Vortrags-Programm zusammengestellt. In jeder Sektion wurde ein Best-Paper-Award (gestiftet von der Deutschen Bank AG) vergeben, dessen Auswahl von einer Jury des Programmkomitees auf Grundlage der Begutachtung der schriftlichen Arbeiten sowie der Bewertung der Präsentationen übernommen wurde.

In Sektion 1 (Virtuelle Geschäftskonzepte und Finanzdienstleistungen im Internet) ging die Auszeichnung an den Bei- trag von Bubl, Visser und Will: „Virtualisierung des Bankgeschäfts". In Sektion 2 (Börsen, Handelssysteme und elektronische Märkte) gab es eine Doppelvergabe für die AMTRAS-Forschergruppe, ein Kooperationsprojekt der Uni Gießen, der Deutschen Börse, Digital und Living Systems, zum Thema „Agentenbasierte Elektronisierung des außerbörslichen Wertpapierhandels" (Gomber, Budimir, Kosciankowski, Urtheil, Lohmann, Nopper, Henning). In Sektion 3 (Ertrags- und Risikomanagement in Banken, Versicherungen und Industrie) wurde der Beitrag von Heyder und Zayer: "Analyse von Kurszeitreihen mit Künstlichen Neuronalen Netzen"ausgezeichnet. Darüber hinaus erhielten Knapp und Hamerle für ihren Beitrag „Multi-Faktor-Modell zur Bestimmung segmentspezifischer Ausfallwabrschein-

Prof. Dr. Hans Ulrich Buhl, Lehrstuhl für Betriebswirtschaftslehre mit Schwerpunkt Wirtschaftsinformatik, Universität Augsburg, D-86135 Augsburg, E-Mail: Hans-Ulrich.Buhl@wiso.uni-augsburg.de; Prof. Dr. Christof Weinhardt, Lehrstuhl für BWL-Wirtschaftsinformatik, Universität Gießen, Licher Str. 70, D-35394 Gießen, E-Mail: Christof.Weinhardt@wirtschaft. uni-giessen.de 
lichkeiten für die Kredit-Portfolio-Steuerung" einen Preis für ihre innovative wissenschaftliche Arbeit zu einem hochaktuellen Praxisproblem.

Im Rahmen der Tagung warteten drei besondere Highlights im Hause der Deutschen Börse auf die Teilnehmer: zunächst präsentierte Herr Frank Gerstenschläger (Mitglied des Vorstands der Deutsche Börse Systems) Xetra Release 3, das wenige Tage später erfolgreich in Produktion ging, und warf einen Blick in die Zukunft von elektronischen Handelssystemen eine gelungene Einstimmung auf das Thema der anschließenden, z. T. kontrovers geführten und spannenden Podiumsdiskussion zum Thema „Die Rolle von Banken, Brokern und Börsen in Elektronischen Finanzmärkten“. Diese wurde von Hermann Kutzer (Chefredakteur der Finanzzeitung des Handelsblattes) moderiert und war mit äußerst kompetenten Teilnehmern besetzt: Dr. Jörg Franke (Mit- glied des Vorstands, Deutsche Börse), Dieter Heinemann (Vorstand ICF-Kursmakler AG), Frank Mattern (McKinsey), Thomas Müssener (Head of Systems, Global Markets, Dresdner Bank), Holger Timm (Geschäftsführer Berliner Freiverkehr) und Prof. Dr. Hartmut Schmidt (Universität Hamburg).

Das dritte Highlight war eine Einladung des Gastgebers auf das Parkett der Deutschen Börse, über dessen zukünftige Bedeutung zuvor sehr ausführlich diskutiert wurde. Das Ambiente und der gelungene Festempfang - untermalt mit Jazz-Musik der Band Tobasco - bot einen hervorragenden Rahmen für anregende, informelle Gespräche.

Eingeladene Vorträge rundeten die Vortragssessions mit aktuellen Beiträgen aus der Praxis ab. Höhepunkte der Referate waren der Eröffnungsvortrag von Herrn Prof. Dr. Jochum (GEFM) zum Thema „Banking im Informationszeitalter“ sowie der Abschlußvortrag zum „Banking nach dem Jahr 2000: Marktstrukturen, Merger und die Konsequenzen für das ITManagement" von Herrn Dr. Hans-Gert Penzel (HypoVereinsbank AG). Es freut uns, daß Herr Dr. Penzel bereit war, zu seinem Vortragsthema auch einen Beitrag für dieses Schwerpunktheft auszuarbeiten.

Wir hoffen, mit den ausgewählten Beiträgen eine gute Mischung aus Praxis und Forschung sowie aus IT und Finanz-/Bankwirtschaft gefunden zu haben, wünschen den Lesern eine anregende Lektüre und freuen uns über jede Art der Resonanz, die wir auch gerne in der Rubrik Meinung/ Dialog publizieren.

P.S. zum Vormerken: Aufgrund der positiven Resonanz steht der nächste Termin bereits fest: Die dann integriert durchgeführte Tagung WI/IF 2001 findet vom 19.-21. September 2001 in Augsburg/ München statt. 